\title{
INTERNATIONALISMEN AUS LINGUISTISCHER UND DIDAKTISCHER PERSPEKTIVE
}

\author{
Dr. Diana Babušytė \\ General Jonas Žemaitis Militärakademie Litauens \\ Mykolas Romeris Universität
}

\begin{abstract}
Annotation. Der vorliegende Beitrag setzt sich mit dem Thema ,Internationalismen “ auseinander. Hier wird ein Überblick über die Internationalismenforschung gegeben. Ein besonderes Augenmerk wird dabei auf die Klassifikation und Typologie fremdsprachlicher Übernahmen gelegt, die auch im Lernprozess ihre Anwendung finden können. Da die Internationalismenforschung nach Gemeinsamkeiten zwischen verschiedenen Sprachen der Welt sucht, haben ihre Erkenntnisse auch einen festen Platz in der Fremdsprachendidaktik. Darüber hinaus werden die didaktischen Implikationen des Phänomens ,Internationalismus “ diskutiert und es wird gezeigt, wie internationale Lexik im Fremdsprachenunterricht eingesetzt werden kann.
\end{abstract}

Schlüsselwörter: Globalisierung, Internationalismen, kontrastive Linguistik, Fremdsprachendidaktik, Tertiärsprachendidaktik.

\section{Einleitung}

Wir kennen alle den Mythos über die babylonische Sprachverwirrung, über die Entstehung der Sprachenvielfalt, den Turmbau zu Babel. Wie viele Sprachen gibt es auf der Welt? Laut modernen Sprachforschern zählt man nämlich etwa 7000 Sprachen, Dialekte nicht mit eingerechnet. Verschiedene Sprachen kommen öfter und intensiver in Kontakt. Als Folge dieser Kontakte kommt es zu gegenseitiger Beeinflussung und zu Entlehnungen lexikalischen Materials. Die Menschheit wächst dadurch immer mehr zusammen. Wir reisen viel, wir lernen neue Sprachen und international verbreitete Wörter sind für jeden von uns eine Erleichterung, sowohl auf Reisen als auch im Lernprozess.

Zur Aktualität des vorliegenden Beitrags: Alle Ähnlichkeiten zwischen Sprachen, die uns das Erlernen einer Fremdsprache erleichtern, sind große Hilfen im Fremdsprachenerwerb. Beim Erlernen der zweiten Fremdsprache greifen wir auf die Erfahrungen der davor erlernten Fremdsprache zurück, wobei die Muttersprache die Grundlage bildet. Jeder Lerner sucht (bewusst oder unbewusst) nach den sogenannten „Transferbrücken“: „Welche Elemente, Einheiten, Strukturen 
der Muttersprache (L1) und der ersten Fremdsprache (L2) können mit den vergleichbaren Elementen, Einheiten und Strukturen der Tertiärsprache (L3) in Beziehung gesetzt und verbunden werden?" (Neuner, 2003: 25). Beim Bau dieser Transferbrücken können u.a. Internationalismen, Lehnwörter sowie ähnliche grammatische Strukturen eine wichtige Rolle spielen.

Den Untersuchungsgegenstand des vorliegenden Beitrags bilden Internationalismen. Das Hauptziel der Arbeit liegt darin, das Phänomen „Internationalismus“ aus linguistischer und didaktischer Sicht zu betrachten. Für die Verwirklichung dieses Ziels sind folgende Aufgaben zu erfüllen: Im ersten Teil wird ein Überblick über die Internationalismen- sowie Fremdwortforschung gegeben, indem verschiedene Typologien von fremdsprachlichen Übernahmen in der Germanistik und Lituanistik dargestellt werden. Im nächsten Teil werden Internationalismen im Lichte der Tertiärsprachendidaktik diskutiert. Hier werden die wichtigsten Ansätze im Fremdsprachenunterricht diskutiert, die auf dem kontrastiven Vergleich von (indogermanischen und nicht-indogermanischen) Sprachen hauptsächlich auf der Grundlage der Lesekompetenz basieren. Dabei wird die deskriptiv-analytische Methode verwendet.

\section{Internationalismen aus linguistischer Perspektive}

Die Beschäftigung mit dem Einfluss anderer Sprachen bzw. mit Lehn- und Fremdwörtern ist kein neuer Untersuchungsgegenstand in der Linguistik. Das Thema zählt bereits zu den klassischen Fragen der Sprachforschung. Die Debatte spielt sich hauptsächlich zwischen zwei Standpunkten ab: Der Betonung der Vorteile von lexikalischen Übernahmen aus einer Lingua franca einerseits und der Kritik daran andererseits. Kristin Otto (2009: 6) gibt in ihrer Arbeit Zitate von zwei großen Germanisten (Johann Wolfgang Goethe und Jakob Grimm) wieder, die diese unterschiedlichen Standpunkte sehr treffend präsentieren. J. W. Goethe beschäftigte sich mit der Problematik der Fremdwörter, die er als Bereicherung für die Muttersprache empfand. Das wird in folgendem Zitat deutlich:

„Die Gewalt einer Sprache ist nicht, daß sie das Fremde abweist, sondern daß sie es verschlingt" (Goethe, 1981: 508).

Jakob Grimm betrachtet dagegen das fremde Wortgut eher kritisch mit sogar puristischen Schattierungen:

„Alle Sprachen, solange sie gesund sind, haben einen Naturtrieb das Fremde von sich abzuhalten und wo sein Eindrang erfolgte, es wieder auszustoßen, wenigstens mit den heimischen Elementen auszugleichen. [...] Fällt von ungefähr ein fremdes Wort in den Brunnen einer Sprache, so wird es solange darin umgetrieben, bis es ihre Farbe annimmt und seiner fremden Art zum Trotze wie ein heimisches aussieht. [...] Es ist Pflicht der Sprachforschung und zumal eines deutschen Wörterbuchs dem maßlosen und unberechtigten Vordrang des Fremden Widerstand zu leisten" (Grimm 1854, XXVI). 
In vielen gemeinsprachlichen Wörterbüchern kann man eine Internationalismus-Definition finden: Zusammenfassend könnte man sagen: Ein Internationalismus ist „ein Wort, das mit gleicher oder ähnlicher Ausducksseite und mit gleicher oder ähnlicher Bedeutung in verschiedenen Sprachen verbreitet und oft ohne Übersetzung verständlich ist" (Turska, 2009: 50; zit. nach Volmert, 1996: 220). Kaum ein linguistisches Wörterbuch gibt eine eindeutige und klare Definition des Terminus Internationalismus. Es gibt wenige wissenschaftliche Abhandlungen, die diesem Thema gewidmet sind. Braun, Schaeder und Volmert (1990: Vorbemerkung) nennen die Internationalismenforschung „terra incognita“, die jeweils einer anderen Disziplin (allgemeiner und vergleichender Sprachwissenschaft, kontrastiver Linguistik, Fremdwortlexikologie, Universalienforschung) zur Erkundung überantwortet wurde, deswegen waren die Internationalismen lange kein Gegenstand linguistischen Interesses.

Peter Braun gilt als Wegbereiter der Internationalismen-Forschung in Westdeutschland. 1990 erschien in der Reihe Germanistische Linguistik der erste Sammelband von Peter Braun, Burkhard Schaeder und Johannes Volmert Internationalismen - Studien zur interlingualen Lexikologie und Lexikographie, der ein beträchtliches Echo in der Germanistik fand. Die Mehrzahl der Rezensionen des ersten Bands zeigte deutlich, dass ein großes Interesse an der Thematik bestand. Aus diesem Grund entstand Mitte der 90er Jahre die Idee, den zweiten Sammelband folgen zu lassen. Das Anliegen der Herausgeber war v. a. jungen Wissenschaftlern eine Gelegenheit zu geben, ihre Forschungsergebnisse im Rahmen von Dissertationsprojekten zu publizieren sowie auch Beiträge zu finden, die sich mit dem Vorkommen von Internationalismen in bisher nicht berücksichtigten Vergleichssprachen, darunter auch nicht-indoeuropäischen Sprachen, beschäftigten. So erschien 2003 der zweite Band von Braun, Schaeder und Volmert Internationalismen II. - Studien zur interlingualen Lexikologie und Lexikographie mit neuen Anstößen in dieser Forschungsrichtung. Er führte das fort, was die Autoren des ersten Sammelbandes vor mehr als 20 Jahren begonnen hatten.

Im Bereich der Typologie werden verschiedene Kombinationen bzw. Typen von formaler Übereinstimmung $(+)$ und Nichtübereinstimmung (-) potenzieller Internationalismen vorgeschlagen. Die Übereinstimmung auf der inhaltlichen Ebene wird als Äquivalenz und die Übereinstimmung auf der formalen Seite wird als Kongruenz bezeichnet. Verallgemeinert könnten folgende Typen von Internationalismen unterschieden werden (Volmert, 1990; Özen, 1999; Schaeder, 2003; Babusytė, 2017):

Typ 1.: eine sehr hohe Übereinstimmung auf phonetischer und auf graphemischer Ebene, z. B. dt. Festival, Fiasko; frz. festival, fiasco; engl. festival, fiasco etc. Hierbei handelt es sich um die sog. Fremdwörter, die in ihrer fremdsprachlichen Variante fast unverändert in die Nehmersprache übernommen werden.

Typ 2.: weitgehende graphemische Übereinstimmung mit erheblichen 
Abweichungen in der phonetischen Realisierung, z. B. dt. Figur, feudal; frz. figure, féodal; engl. figure, feudal etc.

Typ 3.: weitgehende phonetische Übereinstimmung mit unterschiedlicher graphemischer Realisierung, z. B. dt. Fassade; frz.facade; engl. facade; russ. фacad etc. Hier geschieht die Integration ausschließlich auf der graphemischen Ebene. Es handelt sich dabei um eine Substitution der in den entsprechenden Wörtern vorkommenden Fremdgrapheme mit dem Ziel, die quellsprachige phonologische Form weitgehend zu erhalten. Diese wird durch eine indigene Schreibung gestützt, wobei dadurch die graphematische Identität von Fremdwort und quellsprachigem Muster aufgegeben bzw. eingeschränkt wird, z. B. ital. frappè - lit. frapè; span. bachata - russ. бayama etc.

Typ 4: fließende Übergänge zwischen Kongruenz und Nicht-Kongruenz (sowohl in graphemischer und phonetischer als auch in morphologischer Hinsicht), z. B. dt. fixieren, frankieren; frz. fixer, affranchir; span. fijar, franquear; engl. fix, frank; russ. фиксировать, франкировать. Bei diesem Typ geht es um fremdsprachliche Übernahmen, die im Wesentlichen an die untersuchten Sprachen morphologisch (auch graphemisch und grapho-phonemisch) angepasst worden sind. Das wären in der klassischen Terminologie Lehnwörter, die phonetisch, graphisch und morphologisch integriert sind, z. B. lit. prezidentas, engl. discrimination, dt. schockieren, russ. демонстрировать.

Internationalismen gehören zum Phänomen „lexikalische Entlehnung“. Verschiedene Versuche zur Klassifizierung der Entlehnungen in der Sprachenwissenschaft haben zu einer verzweigten und nicht immer eindeutigen Terminologie geführt. Eine Menge von Arbeiten auf diesem Gebiet schafft Verwirrung, aber hier wird sich auf die wichtigsten Typen konzentriert, die möglicherweise auch im Lernprozess von Bedeutung wären. Die von Betz im Jahre 1959 geprägte Terminologie zur Lehngutforschung wird als wertvolle Basis für sprachwissenschaftliche Untersuchungen von fremdsprachlichen Übernahmen benutzt, obwohl sie mehrfach modifiziert wurde (z. B. Munske, 2004; Altleitner, 2007; Bußmann, 2008; Burmasova, 2010 etc.). Man trennt zwischen äußerem (formbezogenen) und innerem (inhaltsbezogenen) Lehngut. $\mathrm{Zu}$ dem äußeren Lehngut gehören folgende Typen:

Lehnwörter: Damit werden fremdsprachliche Übernahmen bezeichnet, die sich im Wesentlichen in Aussprache, Schreibung und Form an das System der Nehmersprache angepasst haben. Sie sehen wie einheimische Wörter aus, z.B. lat. formula - dt. Formel; franz. bureaucratie - russ. бюрократия; ital. tenor - finn. tenori, engl. lypossage - 1t. liposãžas etc.

Fremdwörter: Das sind Wörter, die in ihrer fremdsprachlichen (lateinischen, griechischen, französischen) Variante unverändert in die Nehmersprache übernommen werden. Es handelt sich hier um eine sehr hohe Übereinstimmung sowohl auf phonetischer als auch auf graphischer Ebene, z.B. dt. Renaissance, Baby, Jeans, Interview, Festival, Reggae, Dessert, Prosseco im Deutschen. 
$\mathrm{Zu}$ dem inneren Lehngut (Lehnbildungen) gehören Lehnübersetzungen (Glied-für-Glied Übersetzungen, z.B. engl. skyscraper - lit. dangoraižis, engl. body art - 1t. kúno mẽnas, engl. outsider - dt. Außenseiter; ital. espresso doppio - russ. двойной эспрессо); Lehnübertragungen (freiere Reproduktion des Vorbilds, wobei ein Glied wörtlich und das andere frei übertragen wird, z.B. engl. headphones - dt. Kopfhörer, frz. art informel - lit. befòrme dailẽe, engl. jazzman - russ. джазовый музыкант) und Lehnschöpfungen (formal unabhängige Neubildung, z.B. engl. fiftyfifty - dt. halbe-halbe; engl. air conditioning - dt. Klimaanlage; engl. brainstorm lit. minčiu lietùs etc.).

In der Lituanistik gibt es eine ähnliche Auffasssung von den Begriffen Lehnwort und Fremdwort. Die Werke von Palionis (1999), Jakaitienè (1980), Rudaitienè / Vitkauskas (1998) sind die bekanntesten Arbeiten auf diesem Gebiet. Nach dem Assimilationsgrad werden folgende Typen des fremdsprachlichen Wortgutes unterschieden:

Echte Entlehnungen (völlig in die Nehmersprache integrierte Wörter; allgemein bekannt als Lehnwörter, z. B. batas, knyga, botagas, agurkas, dvaras, grybas etc.);

Internationalismen (in vielen Sprachen verbreitete Wörter, deren Assimilationsgrad geringer als bei den Lehnwörtern ist; sie sind in das phonetische und grammatische System der Nehmersprache integriert, werden aber als Wörter fremdsprachlicher Herkunft empfunden, z. B. aeroportas, atomas, kostiumas, demokratija, fejerverkas, kosmosas, mitingas, serenada etc.);

Fremdwörter/Fremdheiten: (Wörter, die neben den einheimischen existieren, der gemeinsamen Sprachnorm nicht entsprechen und deren fremde Herkunft offensichtlich ist, z. B. bulka, biednas, nariadas, gaspadorius, spravka, naboika etc.) (vgl. Jakaitienè, 1980: 57-64; Rudaitienè, Vitkauskas, 1998: 11).

Unter einem Internationalismus werden in der Lituanistik oft Wörter lateinischer oder griechischer Herkunft verstanden, oder auch Wörter, die aus der heutigen Lingua franca - dem Englischen - verbreitet sind und gleichzeitig lateinisch-griechischen Ursprung haben (Vaicekauskienè, 2004: 12). Die Autorin weist auf solche Sprachwissenschaftler wie Gundersen (1997) und Thomas (1991) hin, die ihrerseits die Meinung vertreten, dass Französisch oder Englisch weniger zu der Entstehung der Internationalismen beitragen und erst das Lateinische die wichtigste Hauptquelle dafür ist. Dagegen ist kritisch einzuwenden, dass andere Linguae francae außer Acht gelassen werden, die enorme Spuren in vielen Sprachen hinterlassen und zur Bereicherung ihrer Wortschätze gedient haben. Die internationale und europäische Sprachgeschichte drückt sich in den folgenden großen Entlehnungsbewegungen aus, die zur Entstehung und Verbreitung von Internationalismen geführt haben: Französisch (Allee, Mode, Dessert, Mayonnaise, Kognak etc.); Italienisch (Aquarell, Arie, Pizza, Espresso, Spaghetti etc.); Englisch (Manager, Disko, Hit, Jazz, Show etc.); Russisch (Steppe, Tundra, Datscha, Babuschka, Pirogge etc.); Arabisch (Alkohol, Jasmin, Kaffee, Zucker, Algebra 
etc.); Japanisch (Sumo, Karate, Sushi, Karaoke, Tsunami etc.); Türkisch (Harem, Döner, Yoghurt etc.); skandinavische Sprachen (Fjord, Saga, Geysir, Sauna etc.); Exotismen (vgl. Otto, 2009: 181-189): Indianische Sprachen (Kajak, Manitu); indische Sprachen (Guru, Yoga, Dharma); Chinesisch (Feng Shui: ,Wind und Wasser') etc.

\section{Internationalismen aus didaktischer Perspektive}

Durch den Vergleich von Sprachen werden einerseits universale und sprachtypologische Aspekte aufgezeigt, die vor allem zu dem Bereich der kontrastiven Linguistik gehören. Andererseits können dabei auch wichtige Ergebnisse für den Fremdsprachenunterricht geliefert werden. Die Beherrschung der Muttersprache (L1) und weiterer bereits gelernter Sprachen (L2, L3) beeinflusst in der Regel das Erlernen jeder Fremdsprache. Diese Beeinflussung kann sowohl Vorteile als auch Nachteile haben. Durch sprachliche Übereinstimmungen können bestimmte Sprachgewohnheiten auf die neue Fremdsprache übertragen werden. In diesem Fall spricht man von einem Transfer. Aufgrund sprachlicher Unterschiede kann jedoch die Übertragung der Sprachgewohnheiten Fehler verursachen, die als Interferenz bezeichnet werden.

In den letzten Jahren wendet man sich verstärkt der Erforschung der sog. Tertiärsprachendidaktik zu, derer folgende Aspekte besondere Berücksichtigung und konzeptionelle Erweiterung finden:

1. kognitives Lernen, das sich durch den Sprachvergleich auf die Entfaltung der Sprachbewusstheit konzentriert. Dabei wird der Muttersprache und der Sekundärsprache die Rolle des „Türöffners“ zum Sprachbewusstsein zugeschrieben (vgl. Neuner, 2009: 4).

2. die Entwicklung der Sprachlernbewusstheit, die sich vor allem durch die Einübung und Erweiterung von Lerntechniken und Lernstrategien auszeichnet.

3. die Inhaltsorientierung, die hauptsächlich altersgemäße Inhalte und die eigene Welt der Lernenden einbezieht.

4. die Textorientierung, die z. B. auf der Entwicklung von Verstehens- und Lesestrategien sowie auf dem Einbezug von zeitgemäßen Textsorten basiert.

Der kontrastive Vergleich sowie die sog. internationalen Wörter sind unmittelbar mit der Entfaltung der Sprachbewusstheit und der Textorientierung verbunden. Die Entfaltung der Sprachbewusstheit ist einer der wichtigsten Grundsätze der Tertiärsprachendidaktik. Er wird auf das Anknüpfen an die bereits vorhandenen Mutter- und Fremdsprachenkenntnisse und auf den intersprachlichen Vergleich gerichtet. Dabei werden in erster Linie sprachliche Ähnlichkeiten zwischen den Sprachen (L1, L2 und L3) bewusst gemacht bzw. hervorgehoben (vgl. Vicente, Pilypaitytė, 2015: 54). In den DaF-Lehrwerken kann man eine Menge Bespiele im Bereich der Lexik finden, innerhalb derer sich die Parallelen bzw. Ähnlichkeiten vorwiegend auf folgende Phänomene stützen: 
a) die internationale Lexik bzw. Internationalismen (z.B. dt. Organisation, Meter, Taxi, Musik, Struktur usw.), ,[...] die zwar in Schreibung und Lautung dem Sprachsystem der jeweiligen Sprache angepasst wurden, deren Bedeutung aber „über die Sprachen hinweg “ gleich geblieben ist" (Kursiša, Neuner, 2006: 4).

b) der Übernahmen aus dem Englischen (Anglizismen als Fremd- und Lehnwörter, z.B. dt. Jeans, PC, Internet, Party, Interview etc.), die ihre Verbreitung vor allem in solchen Bereichen wie Jugendkultur, Sport, Technik, Mode, Medien oder Gastronomie fanden.

Die Entwicklung von Sprachbewusstsein soll von Anfang an gefördert werden, deswegen erfolgt der Einsatz solcher lexikalischen Parallelen meistens in den ersten Unterrichtseinheiten, um die Lernenden zu ermutigen und ihnen bewusstzumachen, dass sie schon am Anfang des Fremdsprachenlernens im Stande sind, viele Wörter zu erkennen und einfache Sätze sowie kurze Texte zu verstehen. Dabei hilft die Aktivierung der Vorkenntnisse aus ihrer Muttersprache und einer anderen bereits gelernten Fremdsprache. Dies führt zu ersten Erfolgsergebnissen beim Deutschlernen und stärkt die Motivation der Lernenden (vgl. Łyp-Bielecka, 2016: 183).

Nehmen wir z. B. die Fremdsprachenkonstellation Deutsch-Englisch. Durch das breite gemeinsame Sprachpotenzial, vor allem im Wortschatzbereich kann im Anfangsunterricht im Verstehensbereich schnell und effizient ein relativ großer Grundwortschatz des Deutschen aufgebaut werden. In den heutigen Lehrwerken geht es meistens um Aufgaben, bei denen der Wortschatz aus dem thematisierten Wortfeld gegenübergestellt und verglichen wird. Es führt dazu, dass Lernende die Ähnlicheiten in den Wortschätzen der beiden Sprachen erkennen und sich die deutschen Vokabeln schneller merken. Bei solchen Aufgaben kann auch auf die Unterschiede in der Schreibung und Aussprache hingewiesen werden (vgl. Vicente, Pilypaityte, 2015: 54).

Das Prinzip der Textorientierung entsteht aus der Verstehens- und Inhaltsorientierung. Die sog. „fremde Welt" soll im Fremdsprachenunterricht vor allem mediengebunden präsentiert werden, z.B. in Lese- und Hörtexten, Bildern, Videos, Filmen etc. (vgl. Kursiša, Neuner, 2006: 6). Deswegen nimmt die Arbeit mit Lesetexten von Anfang an einen wichtigen Platz im Tertiärsprachenunterricht ein. Die sog. L3-Textdidaktik bezieht sich auf die Arbeit mit synthetischen Texten:

a) Text zu einem Wortfeld erarbeiten: Die Aufgaben sollen die Lernenden zum selbständigen Arbeiten anleiten, z.B. sollen sie einen deutschen Text mit vielen Internationalismen (Fremd- Lehnwörtern) global verstehen und erkennen können, dass nicht jedes einzelne Wort eines Textes übersetzt werden muss, um seinen Sinn zu erfassen. Hier ist ein Beispieltext aus dem Lehrwerk studio $d$ (Kurs- und Übungsbuch, S.28), der darauf hinzielt, dass die Lernenden ein Text zu Wortfeldern (Kaffee, Geografie, andere) erarbeiten sollen:

Das Kaffeetrinken ist eine arabische Tradition. Die Türken haben Mokka international populär gemacht. In Europa hat Österreich eine lange 
Kaffeehaustradition und viele Kaffeevariationen. Heute ist Kaffeetrinken „, in “. Caffé Latte, Espresso und Cappuccino heißen die Top-Favoriten in Hongkong, New York, Berlin und St. Petersburg. Café-Ketten wie Starbucks, Segafredo und Coffee Bean sind so international wie McDonalds. Cafés sind ideal für die Kommunikation und für Kontakte.

b) b) (Englisch-deutsche) Paralleltexte: Mit Hilfe der sog. „Paralleltexte“, die vor allem viele Internationalismen erhalten, kann der deutsche Text selbständig durch paralleles Lesen in seiner globalen Bedeutung erfasst werden. Durch die vergleichende Analyse von derartigen synthetischen „Paralleltexten“ können die Lernenden dazu angeregt werden, sich die Sprachsysteme im Bereich von Wortschatz und Grammatik selbständig zu erschließen (vgl. Kursiša, Neuner, 2006: 7). Hier ist ein Beispiel für einen Paralleltext (Marx, 2008):

Die Geschichte des Bieres beginnt vor 6000 Jahren, als die Sumerer den Gärungsprozess entdeckten. Die Kunst des Bierbrauens stammt von den Babyloniern und den Ägyptern und durch sie erreichte das Bier auch die Römer. Die nannten es ein barbarisches Getränk.

The history of beer begins 6000 years ago, when the Sumerians discovered the fermentation process. The Babylonians and Egyptians developed the art of beer brewing and through them, beer reached the Romans. They called it a barbaric drink.

Das wichtigste Ziel der Entwicklung des Leseverstehens ist auch der kompetente Umgang mit authentischen fremdsprachlichen Texten. Die Autoren von gegenwärtigen DaF-Lehrwerken finden solche authentischen Texte vor allem im Internet. Für den erfolgreichen Lernprozess erhalten sie einen hohen Anteil an Wörtern aus dem gemeinsamen germanischen Wortschatz, an Internationalismen und an Lehnwörtern aus dem Englischen. So können sie in ihrer globalen Bedeutung relativ leicht erschlossen werden. Die Arbeit mit authentischen Texten zielt auf das globale Leseverstehen hin. Hier ist ein Beipiel für einen authentischen Text (Kaewwipat, 2012):

London. Superstar Madonna steht in Verhandlungen für eine große WeltTournee in diesem Sommer. Wie die 2Sun "erfahren hat, will die US-Sängerin ihre Tour im Juni in San Francisco starten. Nach mehreren Konzerten in den USA sollen Auftritte in Europa folgen. Selbst Israel stehe auf dem Tourplan. Madonna hatte ihr letztes Live-Konzert vor drei Jahren auf der „Drowned World Tour" gegeben. Danach beschränkte sich die zweifache Mutter auf TV-Auftritte wie bei den „MTV Video Music Awards" im August des vergangenen Jahres mit Britney Spears und Christina Aquilera.

Den meisten wissenschaftlichen Forschungen und didaktischen Ansätzen ist gemeinsam, dass sie sich hauptsächlich innerhalb des indoeuropäischen Sprachraums bewegen. Wie sieht es mit nicht-indoeuropäischen Sprachen aus? Können die gewonnenen Erkenntnisse eines kontrastiven Fremdsprachenunterrichts auch für den Erwerb einer strukturell fremderen Sprache genutzt werden? (vgl. 
Specht, 2005: 4).

Wenn wir einen Text in einer nah verwandten Sprache lesen, dann können wir vieles entschlüsseln. Hat man z. B. Kenntnisse in einer romanischen Sprache, so kann man mit vergleichsweise geringem Aufwand Texte in jeder anderen romanischen Sprache relativ leicht verstehen. Der folgende Beispieltext ist ein Ausschnitt aus einer überregionalen Zeitungsmeldung auf Katalanisch. Aufgrund der Internationalismen (z. B. kat. tragèdia / franz. tragédie /dt. Tragödie; kat. persones/ franz. personnes / dt. Personen u.a.) erkennt man schnell, worum es sich in dieser Meldung handelt, u. z. um einen tragischen Flugzeugabsturz über dem Roten Meer (vgl. Specht, 2005: 24):

Tragèdia aèria a Egipte 148 persones, la majoria turistes francesos, moren en caure un avió al mar Roig pocs minuts després d'haver-se enlairat Redacció EL CAIRE. Egipte va viure ahir a la matinada la segona pitjor tragèdia aèria de la seva história, en caure al mar Roig un Boeing 737 amb 148 persones a bord, de les quals 135 eren turistes francesos.

Die Zahl der internationalen Lexeme im Wortschatz indoeuropäischer Sprachen ist sehr hoch. Wenn man die indoeuropäische Sprachfamilie verlässt, so wird der Anteil an gemeinsamen Wortschatz geringer. Specht (2005: 94) weist darauf hin, dass das Türkische beispielsweise überwiegend Übernahmen aus dem Französischen hat und der Anteil an Internationalismen im Wortschatz von türkischen Zeitungen und Zeitschriften relativ hoch, z. B. türk. bisiklet - franz. bicyclette oder türk. sürpriz - franz. surprise. Aber deutlich häufiger kommen internationale Wörter in Fachtexten vor, die zu einem großen Teil auf lateinischer und griechischer Lexik beruhen, z. B. (Specht 2005: 95):

Biyloji bilimlerinin bazı dalları, özellikle morfoloji, fizyoloji, moleküler biyoloji ve genetik, bütün canlılarla ilgilenen genel araştırma alanlarıdır. Oysa ilgisini belirli canlı gruplarryla sınırlandırmış olan botanik, zooloji, mikrobiyoloji, gibi dallar, kendi içlerinde de altdallara ayrılarak iyice özelleşmişstir. Örneğin zoolojinin bir altdalı olan ornitoloji yalnız kuşları, entomoloji böcekleri inceler. Botaniğin altdallarından algolojinin konusu suyosunları (algler), mikolojininki mantarlardrr. Milyonlarca tekhücreli canlry konu alan mikrob iyoloji de yalniz bakterileri inceleyen bakteriyoloji ve virüsleri inceleyen viroloji gibi altdallara ayrılmıştır.

Anhand des internationalen Wortschatzes lassen sich durch kontrastive Vegleiche spezifische Schreib- und Ausspracheregeln des Türkischen und anderen L1/L2/L3-Sprachen thematisieren, z. B. türk. biyloji-franz. biologie - dt. Biologie; türk. virüs - franz. virus - dt. Virus etc. Eine Eigenschaft des Türkischen, die nach dem Lesen dieses Textes auffällt, ist die sogenannte Vokalregel: Im Türkischen stehen keine zwei Vokale nebeneinander, sie werden durch einen Bindekonsonant getrennt, z. B. bakteriyoloji, fizyoloji. 


\section{Fazit}

Große Entlehnungsbewegungen aus verschiedenen Linguae francae sind die Hauptquellen zur Entstehung von lexikalischen Gemeinsamkeiten aller Teile der Welt. In unserer globalisierten Welt erleichtert dieses Phänomen das Zusammenleben verschiedener Kulturen. Darüber hinaus wird der internationalen Lexik immer mehr Beachtung geschenkt, sowohl in sprachwissenschaftlichen Abhandlungen, als auch in didaktischen Ansätzen.

Unterschiedliche mehrsprachigkeitsfördernde Aufgaben- und Übungsformen, die auf den Prinzipien der Tertiärsprachendidaktik basieren, finden immer öfter Eingang in die Lehrbücher. Dieses Potenzial kann im Lehrprozess genutzt und angemessen eingesetzt werden, denn alle Ähnlichkeiten zwischen Sprachen, die dem Lerner den Erwerb einer weiteren Fremdsprache (sowohl indogermanischer als auch nicht-indogermanischer Sprache) erleichtern, sind willkommene Hilfen im Fremdsprachenunterricht. Der positive Einstieg in die Fremdsprache bietet sich $u$. a. mithilfe von internationalen Wörtern an.

Internationalismen ermöglichen nicht nur die Orientierung in einer fremdsprachigen Umgebung, sondern sind beim Erlernen einer Fremdsprache als Gedächtnishelfer sehr hilfreich. Sie helfen die Unsicherheit vor dem Lernprozess einer Fremdsprache zu überwinden. Vertraute Formen können besonders in der Anfangsphase des Lernens einer Fremdsprache eine große Erleichterung sein und damit eine größere Motivation hervorrufen.

Im Fremdsprachenunterricht können am Beispiel der Internationalismen einzelsprachliche orthographische und morphologische Besonderheiten der erwerbenden Sprache gezeigt und semantische Unterschiede erläutert werden. Für Anfänger und Fortgeschrittene kann die Lektüre von einfachen authentischen oder synthetischen Texten verwendet werden, um Internationalismen zu erkennen oder aktuelle Interlexeme, z. B. nach Wortarten oder semantischen Wortfeldern zusammenzutragen. Beiden FortgeschrittenenkönnenauchliterarischePublikationen oder fachsprachliche Texte eingesetzt werden. Das Erschließen von Regeln durch den systematischen Vergleich wirkt sehr effektiv auf den Fremdsprachenerwerb der Lernenden. Dabei können verschiedene Typologien von fremdsprachlichen Übernahmen bzw. Typen von Internationalismen von Bedeutung sein.

\section{Literaturverzeichnis}

1. Altleitner, M. (2007). Der Wellness-Effekt. Die Bedeutung von Anglizismen aus der Perspektive der kognitiven Linguistik. Frankfurt a.M.: Peter Lang.

2. Babuyte, D. (2017). Internationalismen im Litauischen und Russischen. In: Кременецькі компаративні студіï. Кременець: Гуманітарний факультет Кременецької обласної гуманітарно-педагогічної академії імені Тараса Шевченка, т. 2, Вип. VII, с. 100-109. 
3. Betz, W. (1959). Lehnwörter und Lehnprägungen im Vor- und Frühdeutschen. In: Maurer, F.; Stroh, F. (Hrsg.). Deutsche Wortgeschichte. Bd. 1. Berlin: Schmidt, S. 127-147.

4. Braun, P.; Schaeder, B.; Volmert, J. (Hrsg.). (1990). Internationalismen. Studien zur interlingualen Lexikologie und Lexikographie. Tübingen: Niemeyer.

5. Braun, P.; Schaeder, B.; Volmert, J. (Hrsg.). (2003). Internationalismen II. Studien zur interlingualen Lexikologie und Lexikographie. Tübingen: Niemeyer.

6. Burmasova, S. (2010). Empirische Untersuchung der Anglizismen im Deutschen. Bamberger Beiträge zur Linguistik. Bd. 2. University of Bamberg Press.

7. Bußmann, H. (2008). Lexikon der Sprachwissenschaft. 4., durchgesehene und bibliographisch ergänzte Auflage. Kröner: Stuttgart.

8. Goethe von, J. W. (1981). Maximen und Reflexionen. München: Beck.

9. Grimm, J.; Grimm, W. (1854). Deutsches Wörterbuch. Leipzig: Hirzel.

10. Gundersen, D. (1997). Skriv lånordene på norsk! In: Purisme på norsk, p. 61-64.

11. Jakaitienè, E. (1980). Lietuviu kalbos leksikologija. Vilnius: Mokslas.

12. Kaewwipat, T. (2012). Vortrag zum Thema Mehrsprachigkeit und Mehrsprachigkeitsdidaktik. Online: http://www.nkaewwipat.com/docs/ThK_ Mehsprachigkeit.pdf [abgerufen am 25.02.2018]

13. Kursiša, A.; Neuner G. (2006). Deutsch ist easy! Lehrerhandreichungen und Kopiervorlagen ,Deutsch nach Englisch “für den Anfangsunterricht. Ismaning: Max Hueber Verlag.

14. Łyp-Bielecka, A. (2016). Die Prinzipien der Tertiärsprachendidaktik und ihre Widerspiegelung in den ausgewählten Daf-Lehrwerken für den Anfängerunterricht. In: Glottodidactica XL III/2, S. 177-192. Online: http://pressto. amu.edu.pl/index.php/gl/article/viewFile/6888/6898 [abgerufen am 25.06.2017]

15. Marx, N. (2008). Deutsch nach Englisch, Deutsch mit Englisch. Online: http://www.goethe.de/ins/fr/pro/classesbilangues/Marx_Nicole.pdf [abgerufen am 25.02.2018]

16. Munske, H. H. (2004). Englisches im Deutschen. In: Munske, H. H. (Hrsg.). Deutsch im Kontakt mit germanischen Sprachen. Tübingen: Niemeyer, S. $155-175$.

17. Neuner, G(2003). MehrsprachigkeitskonzeptundTertiärsprachdidaktik. In: Hufeisen, B.; Neuner, G. (Hrsg.). Mehrsprachigkeitskozept-TertiärsprachenlernenDeutsch nach Englisch. Strasbourg: Council of Europe Publishing, S. 13 - 34.

18. Neuner, G. (2009). Mehrsprachigkeitsdidaktikund Tertiärsprachenlernen. Grundlagen - Dimensionen - Merkmale. Online: http://www.hueber.de/sixcms/ media.php/36/Neuner-Mehrsprachigkeitsdidaktik.pdf [abgerufen am 25.02.2018]

19. Otto, K. (2009). Eurodeutsch - Untersuchungen zu Europäismen und Internationalismen im deutschen Wortschatz: eine Arbeit aus der Perspektive der Eurolinguistik am Beispiel von Zeitungen aus Deutschland, Österreich, der Schweiz und Südtirol. Berlin: Logos. 
20. Özen, Ü. (1999). Internationalismen - Konzeption einer interlexikologischen Theorie. Dargestellt am Beispiel der Ergebnisse einer Auswertung von türkischen Tageszeitungen. Universität Siegen. (Dissertation)

21. Palionis, J. (1999). Kalbos mokslo pradmenys. Vilnius: Jandrija.

22. Rudaitienè, V.; Vitkauskas, V. (1998). Vakaru kalbu naujieji skoliniai. Vilnius: Enciklopedija.

23. Schaeder, B. (2003). Neuerlicher Versuch einer theoretischen und methodischen Grundlegung der Internationaslimen-Forschung. In: Braun, P.; Schaeder B.; Volmert J. (Hrsg.). Internationalismen II. Studien zur interlingualen Lexikologie und Lexikographie. Tübingen: Niemeyer, S. 77-107.

24. Specht, T. (2005). Neue Ansätze im Fremdsprachenunterricht für Erwachsene und ihre Anwendung auf nicht-indoeuropäische Sprachen. Eine Untersuchung am Beispiel des Türkischen. Universität Hildesheim. (Magisterarbeit) file://C:/Users/dbabusyte/Downloads/Magisterarbeit_Specht_Juni2005\%20(1). pdf [abgerufen am 25.02.2018]

25. Funk, H. (Hrsg.). (2006). Studio d. A1. Kurs- und Übungsbuch. Berlin: Cornelsen Verlag.

26. Thomas, G. (1991). Linguistic purism. London: Longman.

27. Turska, M. (2009). Internationalismen in der Fachsprache der Gastronomie und Kochkunst im fünfsprachigen Vergleich. Danzinger Beiträge zur Germanistik, Band 28. Frankfurt a.M.: Peter Lang.

28. Vaicekauskienė, L. (2004). Teorinès svetimžodžių prielaidos. Iš: Skoliniai ir bendrine lietuviu kalba: mokslo straipsniu rinkinys. Vilnius: Lietuvių kalbos instituto leidykla, p. 9-29.

29. Vicente, S.; Pilypaitytė L. (2015). Lehrsprachigkeitsdidaktik in Lehrmaterialien. In: Fremdsprache Deutsch 50. S. 52-58. Online: https:// edisciplinas.usp.br/pluginfile.php/2424130/mod_resource/content $/ 5 /$ Vicente $\% 20$ Pilypaityte\%202013.pdf [abgerufen am 25.02.2018]

30. Volmert, J. (1996). Die Rolle griechischer und lateinischer Morpheme bei der Entstehung von Internationalismen. In: Munske, H.H; Kirkness, A. (Hrsg.). Eurolatein. Das griechische und lateinische Erbe in den europäischen Sprachen. Tübingen: Niemeyer, S. 219-235. 


\title{
INTERNATIONALISMS FROM THE LINGUISTIC AND DIDACTIC PERSPECTIVES
}

\author{
Dr. Diana Babušytė \\ General Jonas Žemaitis Military Academy of Lithuania \\ Mykolas Romeris University
}

\section{Summary}

Linguistics and didactics are increasingly focused on international lexis. The phenomenon of international words not only facilitates the coexistence of different cultures but also effectively helps acquiring foreign languages. The object of the work is related to general borrowings of languages, i.e. lexical internationalisms. In linguistics, the term internationalism is frequently identified with such terms as loanword and borrowing. The first part of the article examines the definitions of internationalism presented in various dictionaries and linguistic works as well as gives an overview of the main works in the field of borrowings in the German and Lithuanian linguistics. One of the most important aspects of the work is to discuss the typology of international words as well as to show the types of their integration, which can be used in the process of learning foreign languages.

Internationalisms as political, historical, economic and religious aspects of development are essential elements of lexis of different languages, word formation and usage. They become quickly integrated in languages and enrich them. This common language potential is important in foreign language teaching, whereas all language similarities that help learners to acquire another foreign language (both Indo-European and non-Indo-European) are incentive learning measures.

The second part of the article focuses on didactic issues. The lexemes recognized at the initial stage reduce the feeling of difficulty in the process of learning and stimulate motivation. Particular spelling and morphological subtleties can be explained and the differences of new language semantics can be presented and elucidated by providing students with the examples of international words during the classes of foreign languages. International vocabulary helps beginners to read simple, authentic or synthetic texts, and enables advanced learners to understand literary or specialist texts. Working with international words in texts and systematically comparing them with a mother tongue or another foreign language encourages the development of rules of the new language that is effective for language learners. 


\section{AUTORIAUS LYDRAŠTIS}

Autoriaus vardas, pavardė: Diana Babušytė

Mokslo laipsnis ir vardas: humanitarinių mokslų daktarè

Darbo vieta ir pareigos: Generolo Jono Žemaičio karo akademijos lektorè, Mykolo Romerio universiteto docentè

Autoriaus mokslinių interesų sritys: dabartinė vokiečių kalba, kontrastyvinė lingvistika, eurolingvistika, kalbų tipologija, svetimujų kalbų dėstymo didaktika

Telefonas ir el. pašto adresas: 8 64590727; diana.babusyte@1ka.lt

\section{AUTHOR'S COVER LETTER}

Author's name, surname: Diana Babušyte

Academic degree and name: Doctor of Arts

Workplace and position: General Jonas Žemaitis Military Academy of Lithuania, Lecturer; Mykolas Romeris University, Associate Professor

Author's research interests: modern German, contrastive linguistics, Eurolinguistics, linguistic typology, foreign language teaching methods

Telephone and e-mail address: +370 64590 727; diana.babusyte@lka.lt 